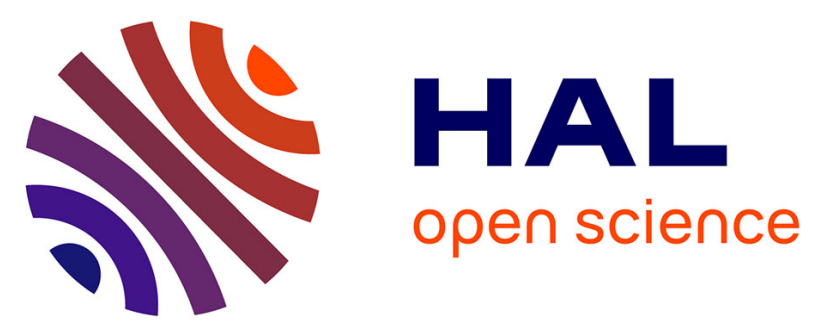

\title{
Sintering mechanisms involved in high-velocity compaction of nascent semicrystalline polymer powders
}

David Jauffres, O. Lame, Gérard Vigier, Florence Dore, Thierry Douillard

\section{To cite this version:}

David Jauffres, O. Lame, Gérard Vigier, Florence Dore, Thierry Douillard. Sintering mechanisms involved in high-velocity compaction of nascent semicrystalline polymer powders. Acta Materialia, 2008, pp.2550-2559. 10.1016/j.actamat.2009.02.012 . hal-00433938

\section{HAL Id: hal-00433938 \\ https://hal.science/hal-00433938}

Submitted on 17 Jan 2022

HAL is a multi-disciplinary open access archive for the deposit and dissemination of scientific research documents, whether they are published or not. The documents may come from teaching and research institutions in France or abroad, or from public or private research centers.
L'archive ouverte pluridisciplinaire HAL, est destinée au dépôt et à la diffusion de documents scientifiques de niveau recherche, publiés ou non, émanant des établissements d'enseignement et de recherche français ou étrangers, des laboratoires publics ou privés.

\section{(ㄷ)(1) $\$$}

Distributed under a Creative Commons Attribution - NonCommercial| 4.0 International 


\title{
Sintering mechanisms involved in high-velocity compaction of nascent semicrystalline polymer powders
}

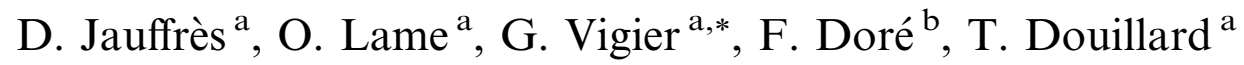

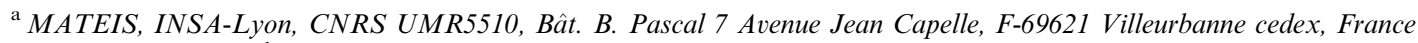 \\ ${ }^{\mathrm{b}}$ CETIM, 7 rue de la presse, BP802, F-42952 Saint Etienne cedex 9, France
}

\begin{abstract}
A new technique, high-velocity compaction (HVC), allows the processing of nascent semicrystalline polymer powders via sintering. For the two polymers studied, polyoxymethylene (POM) and ultra-high molecular weight polyethylene (UHMWPE), it has been observed that during $\mathrm{HVC}$ a fraction of the nascent polymer undergoes melting. After cooling, the material thus comprises both nascent polymer that has not undergone melting and melted-recrystallized polymer. First, the localization of the melted-recrystallized polymer is investigated by microscopic techniques and nanoindentation. This reveals that melting occurs first at particle interfaces. Then, it is demonstrated that welding of the particles occurs via chain diffusion and recrystallization during the cooling. The efficiency of these welding mechanisms is discussed in order to gain an insight into the possibilities of using HVC to process different types of polymers. In addition it is shown that for a given material the overall sintering quality is governed by the fraction of recrystallized material.
\end{abstract}

Keywords: Sintering mechanism; High-velocity compaction; Nascent polymer powder

\section{Introduction}

The success of thermoplastic polymers is largely due to their ability to be processed quickly and cheaply by injection or extrusion molding; however, in some cases these process methods face problems, including the following:

- risk of polymer chemical degradation induced by high processing temperature;

- significant shrinkage and cavity formation during crystallization due to the density difference between crystalline and amorphous phases (e.g. it is nearly impossible to produce thick items made from polyoxymethylene (POM));

- the requirement for low viscosity, leading to the unprocessability of some polymers and limiting the use of fillers (e.g. polytetrafluoroethylene (PTFE) or ultra-high molecular weight polyethylene (UHMWPE)).

\footnotetext{
* Corresponding author.

E-mail address: Olivier.lame@insa-lyon.fr (G. Vigier).
}

"Sintering" processes have been developed to process, for example, UHMWPE [1,2] and PTFE [3,4]. Unlike metals and ceramics, sintering of polymers requires complete melting of the material. Another route to avoid the aforementioned problems consists in using solution process. These processes offer satisfactory solutions to viscosity limitations, but they are long (several hours) and expensive. Moreover, use of solvents is increasingly unacceptable due to environmental considerations. To address shrinkage problems, some attempts have been made to sinter POM [5] and UHMWPE [6] below their melting temperature, but these processes are still under development.

High-velocity compaction (HVC), an innovative process developed in collaboration with CETIM (Technical Center of French Mechanical Industry), allows the processing of polymer powders via sintering [7]. It consists in applying several controlled energy impacts to a heated and powder-filled die (Fig. 1). The die is pre-heated at a temperature called the "processing temperature", $T_{p}$ (first processing parameter). The "total energy" is the second processing parameter and refers to the kinetic energy of one impact 

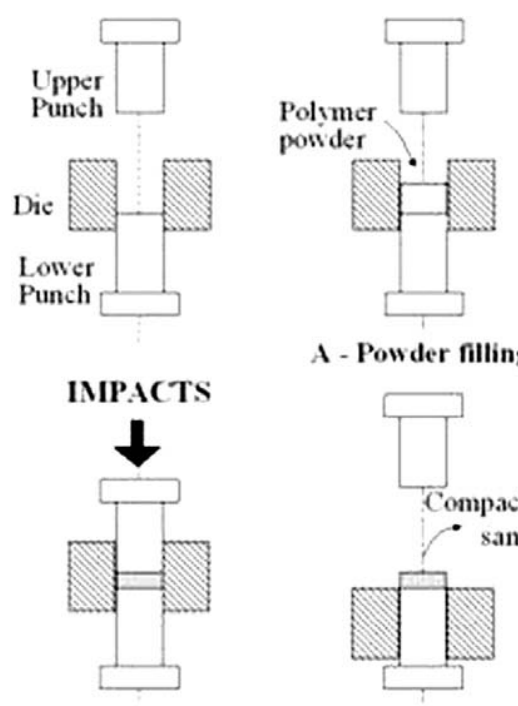

A - Powder filling

B - Compaction

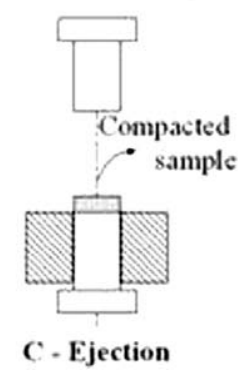

Fig. 1. Schematic representation of the three steps of the HVC process.

multiplied by the number of impacts. The details of the HVC process are described elsewhere $[7,8]$. The energy dissipated in the sample causes its self-heating: temperatures up to $20^{\circ} \mathrm{C}$ above the starting temperature have been measured in samples just after their ejection, which shows that this phenomenon is considerable. The first dozen impacts lead to nearly full densification.

Two semicrystalline polymers, POM and UHMWPE, have been successfully processed by HVC [7,8]. These polymers have been chosen for several reasons, principally because they crystallize easily and exhibit a high degree of crystallinity. Previous studies have shown that the HVC process requires easy crystallizable polymers. Moreover, as noted above, POM and UHMWPE are very difficult to process and HVC provides a suitable alternative approach. Finally, these polymers have very different melt viscosities, and this will help to evaluate the abilities of HVC. Nascent powders, directly extracted from the polymerization reactor, have been used as starting material. These powders have a high degree of crystallinity, which is irreversibly lost after the first melting and thus cannot be attained by crystallization from the melt (i.e. during conventional processing). The physical and mechanical properties of HVC POM and HVC UHMWPE have been studied in detail elsewhere $[7,8]$. The maximum strength is obtained after several high-energy impacts, at a processing temperature $T_{p}$ close to, but below, the polymer melting point.

Due to topological constraints in its amorphous phase, UHMWPE nascent powder exhibits a higher melting peak position than melt-crystallized material during a differential calorimetry scan (DSC) at $10^{\circ} \mathrm{C} \mathrm{min}{ }^{-1}$ [9]. Consequently, the occurrence of a double melting peak for HVC UHMWPE clearly demonstrates the presence of both nascent highly crystalline polymer retained during the process, and melted-recrystallized polymer (Fig. 2 and Ref.

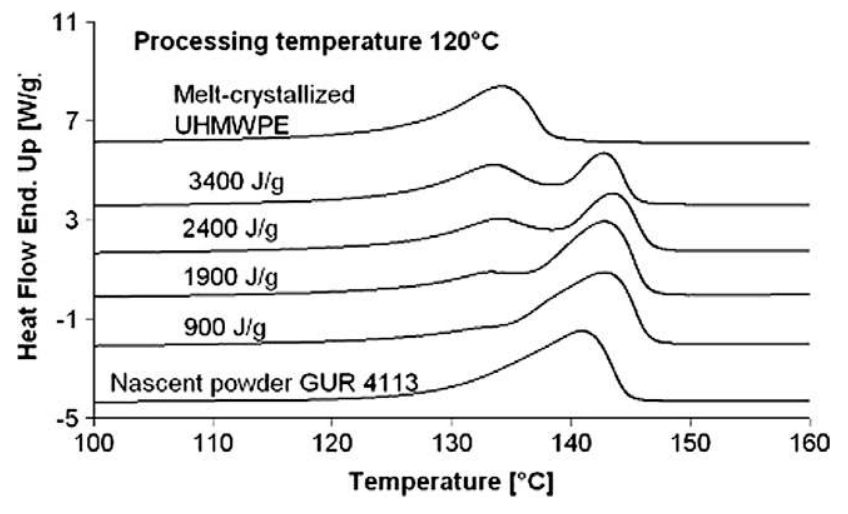

Fig. 2. DSC curves of HVC UHMWPE processed at $120^{\circ} \mathrm{C}$ and total energies ranging from 900 to $3400 \mathrm{~J} \mathrm{~g}^{-1}$. From Ref. [8].

[8]). As nascent polymer recrystallizes with a lower crystallinity after melting, a slight decrease in the crystallinity is also observed, both for HVC POM [7] and HVC UHMWPE [8].

HVC materials exhibit a high elastic modulus due to the partial preservation of nascent high crystallinity. Indeed, it is generally admitted that within the elastic domain, the strain is mainly located in the amorphous phase which is believed to be $10^{2}-10^{4}$ times softer than the crystalline phase, and thus the modulus strongly increases with decreasing amorphous content [10].

At low total energy, the material is brittle with a low fracture stress: powder particles are only poorly welded. On the contrary, at higher total energy, particles are strongly bonded together as proved by the significant ductility of the material [8]. Due to the intrinsic brittleness of nascent powder particles at $20^{\circ} \mathrm{C}, \mathrm{HVC}$ POM is brittle, but its fracture strength is high and the fracture surface is trans-particle, which confirms that a large number of interfaces are welded [7]. For HVC processing, sintering refers to the overall consolidation resulting from local welds of the particles. Therefore, the sintering "quality" depends on the number of welded interfaces and on weld strength.

It has been possible to obtain the fraction of recrystallized material $f_{R}$ from DSC curves by a deconvolution method and from the degree of crystallinty [8]. In Fig. 3, $f_{R}$ is plotted as a function of total energy for different processing temperatures $T_{p}$, specified by their position compared to $T_{m}$, the nascent powder onset of melting peak measured at $10^{\circ} \mathrm{C} \mathrm{min}^{-1}$, i.e. $130{ }^{\circ} \mathrm{C}$ for UHMWPE and $170{ }^{\circ} \mathrm{C}$ for POM. It is observed that $f_{R}$ increases with $T_{p}$ and total energy.

As POM nascent powder is not protected by antioxidants and melted POM has very low viscosity, thermochemical degradation and polymer flowing at ejection limit the processing temperature. Consequently, the maximum recrystallized phase fraction obtained was only about $25 \%$. On the contrary, in the case of UHMWPE, it has been possible to reach large $f_{R}$, up to $90 \%$, since UHMWPE is extremely viscous in the melted state and has a higher resistance to degradation. 


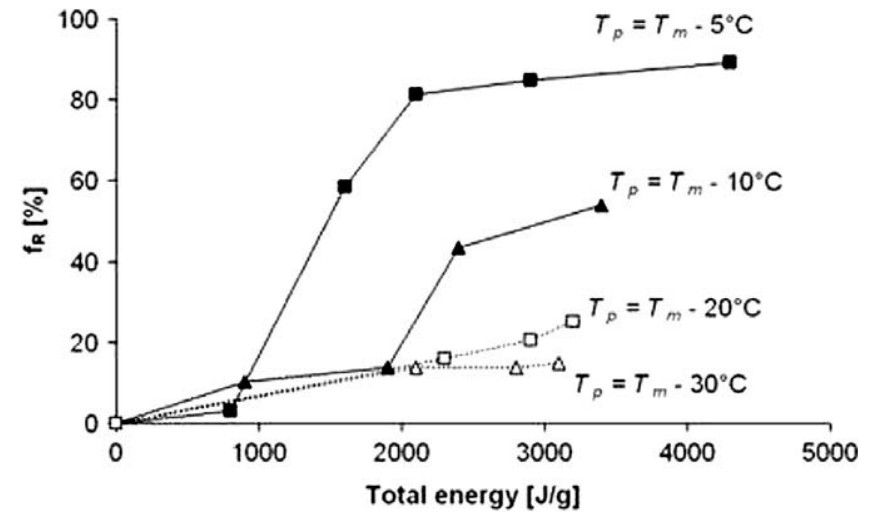

Fig. 3. Recrystallized phase fraction $f_{R}$ of HVC material vs. total energy for different processing temperatures $T_{p}$. Filled symbols refer to HVC UHMWPE while open symbols refer to HVC POM. $f_{R}$ is obtained with an absolute precision of about $10 \%$.

In this paper, electron and optical microscopy, as well as nanoindentation results, bring new evidence for the presence of the recrystallized phase. In addition, they provide an insight into the spatial distribution of the two phases. A detailed review of the possible sintering mechanisms is provided and the overall sintering quality is discussed.

\section{Materials and methods}

\subsection{Low-voltage scanning electron microscopy (SEM)}

HVC material failure surfaces from tensile tests were observed using a FEI XL-30 ESEM field emission gun scanning electron microscope at low accelerating voltage. The use of low voltage $(0.8-1.4 \mathrm{kV})$ allows the observation of non-conducting samples without metal coating and thus the original sample surface characteristics are preserved. This is particularly important for the observation of microfibrils on HVC material failure surfaces. It is worth noting that the electron beam damages the fibrils and that the observation must be performed quickly.

\subsection{Nanoindentation}

Nanoindentation experiments were performed on a Nano IndenterII from MTS Nano Instruments (Oak Ridge, TN). This technique notably allows the concept of hardness to be extended to soft materials and provides a measure of the modulus of elasticity. Furthermore, the instrumented nanoindentation device acts as a mechanical probe down to the nanometer scale. The experiment consists in loading and unloading a surface with a diamond tip and measuring the material response. Flat surfaces of a few $\mathrm{mm}^{2}$ were obtained by cutting samples with a cryoultramicrotome and a glass knife. A specific indentation procedure, coming from our group experience [11], was used to limit the influence of viscoelastic creep. This procedure involves several loading, unloading and holding segments until a fixed depth of $500 \mathrm{~nm}$ and ends with a complete unloading. The loading and unloading segments were performed at $10 \mathrm{~nm} \mathrm{~s}^{-1}$.

The load $P$ is plotted as a function of the displacement $h$, and the slope $S$ of the tangent to the unloading segment is used to evaluate the local Young's modulus $E$ as follows (Fig. 4) [11,12]:

$$
\begin{aligned}
& E_{R}=\frac{\sqrt{\pi}}{2} \frac{S}{\sqrt{A}} \\
& \frac{1}{E_{R}}=\frac{1-v^{2}}{E}+\frac{1-v_{i}^{2}}{E_{i}}
\end{aligned}
$$

where $E_{R}$ is the so-called reduced modulus, $A$ is the contact area between the indenter tip and the material under the maximum load $P_{\max }, v$ is the Poisson's ratio of the tested material, and $v_{i}$ and $E_{i}$ are the Poisson's ratio and the Young's modulus of the indenter tip. $A$ is estimated from the indentation depth and the geometry of the tip (a standard Berkovich nanoindentation tip). A value of 0.4 was used for the POM and UHMWPE Poisson ratios. It should be noted that the choice of Poisson ratio has only slight influence on the calculated modulus [11].

Local Young's modulus values obtained by nanoindentation are generally higher than macroscopic Young's moduli in the case of polymeric materials. Viscoelastic creep during unloading leads to an overestimate of the modulus, even with the use of a specific procedure developed to limit its influence [12].

\subsection{Optical microscopy}

Optical microscopy was used to observe the surfaces before and after nanoindentation experiments. It was performed on a Zeiss Axiophot microscope equipped with a numerical acquisition system. The use of differential interference contrast mode (also called Nomarski contrast), which enhances the perception of the topographym was helpful to visualize the indentation marks, especially in the case of UHMWPE.

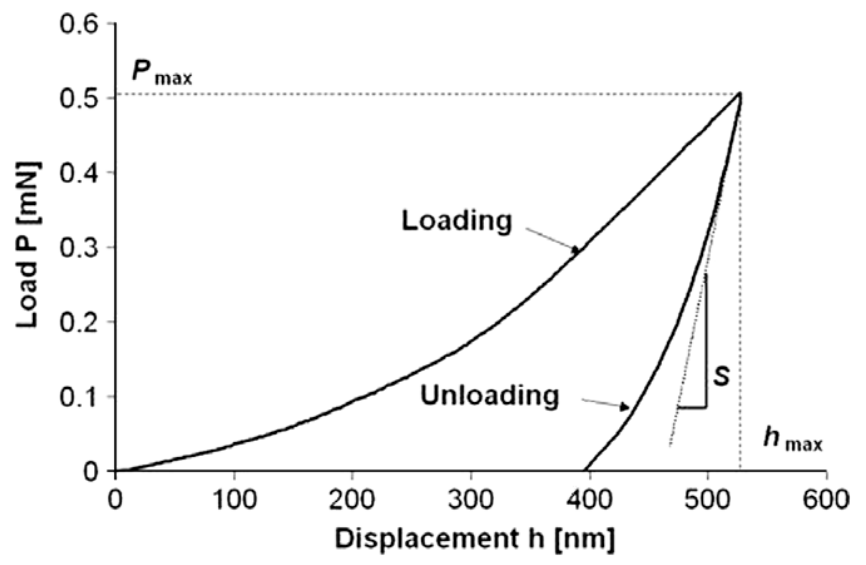

Fig. 4. Typical load-displacement curve obtained during polymer nanoindentation. 


\subsection{Atomic force microscopy (AFM)}

AFM was performed to observe the relief of the cryoultramicrotomed surfaces. A Veeco NanoScope Dimension 3100 (Santa Barbara, CA) was used in tapping mode with tips oscillating in air at a frequency close to the cantilever resonance frequency $(\sim 300 \mathrm{kHz})$.

\section{Results}

\subsection{Two-phase structure investigated by low-voltage SEM}

Our intention was to localize the recrystallized phase, which is believed to be softer than the nascent one due to its lower crystallinity $[7,8,13]$. Fracture surfaces of several HVC materials have been examined under low-voltage SEM. In Figs. 5 and 6, fracture surfaces of HVC POM and HVC UHMWPE with moderate $f_{R}$ are displayed. In both cases, fracture occurs at the particle boundaries, which exhibit numerous fibrils revealing significant local deformations. It is thought that the softer phase, i.e. the

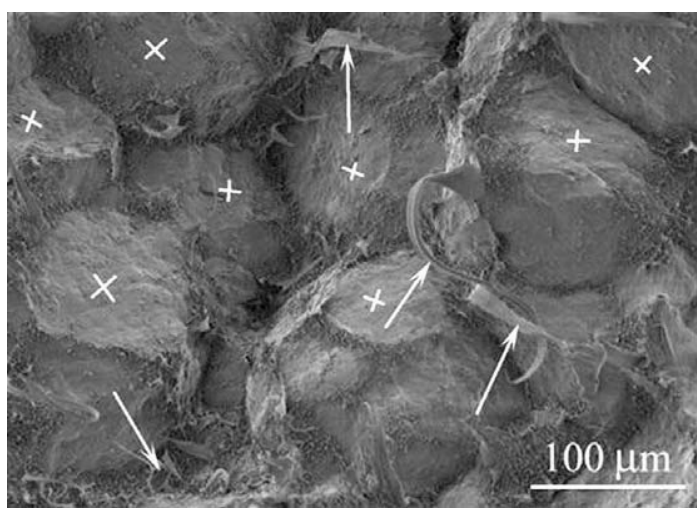

Fig. 5. Interparticle fracture surface of HVC POM from a bending test carried out at $130^{\circ} \mathrm{C}$. Moderate recrystallized phase fraction $\left(f_{R} \sim 20 \%\right)$. Arrows indicate fibrils (recrystallized phase) and crosses indicate nascent particles.

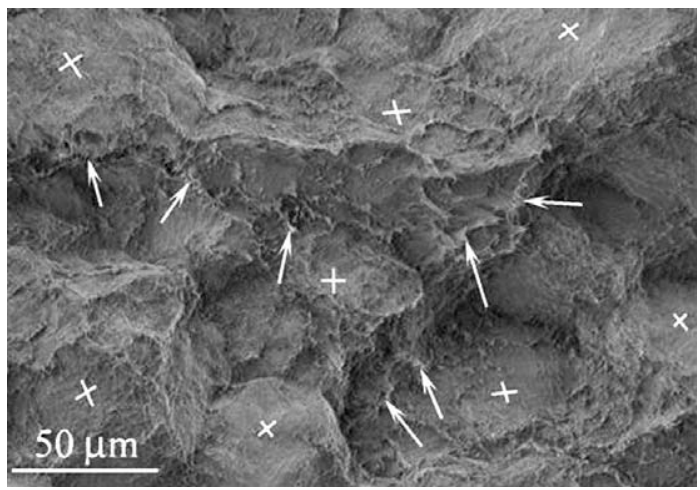

Fig. 6. Interparticle fracture surface of HVC UHMWPE from a tensile test carried out at $20^{\circ} \mathrm{C}$. Moderate recrystallized phase fraction $\left(f_{R} \sim 40 \%\right)$. Arrows indicate fibrils (recrystallized phase) and crosses indicate nascent particles. recrystallized phase, concentrates the deformations and breaks first. It follows that in Fig. 5 and the fibrils correspond to highly stretched recrystallized phase, which suggests that this phase is partially located at particle interfaces.

It should be noted that in the case of HVC POM, during a mechanical test at $20^{\circ} \mathrm{C}$, samples failed in a brittle manner within the nascent phase (trans-particle failure) due to nascent particle intrinsic brittleness [7]. Therefore, in order to allow deformations of the recrystallized phase, the fracture surface (Fig. 5) has been obtained after a mechanical test performed at $130^{\circ} \mathrm{C}$. Indeed, nascent POM particles are no more brittle above the so-called $\alpha_{\mathrm{c}}$ mechanical relaxation attributed to crystalline mobility (around $100-120^{\circ} \mathrm{C}$ at $1 \mathrm{~Hz})$ [7].

Above $f_{R}=50 \%$, the fracture surfaces exhibit thick fibrils, suggesting the presence of large regions of recrystallized material that could correspond to entirely meltedrecrystallized particles (Fig. 7). In this case, the observation of fracture surfaces does not allow us to assess the localization of the phases due to large deformations of a significant fraction of the material.

\subsection{Two-phase structure investigated by optical microscopy and nanoindentation}

In light of the fracture surface analysis, it is thought that a part of the recrystallized polymer is located at particle interfaces at low $f_{R}$. Nanoindentation was performed to attempt to obtain more direct evidence of recrystallized phase localization. As already mentioned, the recrystallized phase should be softer than the nascent phase owing to its lower crystallinity. For UHMWPE, it has been shown that there is a linear dependence of the macroscopic elastic modulus on the recrystallized phase fraction $f_{R}$. Assuming that HVC UHMWPE recrystallized phase elastic modulus is equal to that of conventionally processed UHMWPE, an extrapolation has allowed us to estimate the nascent phase elastic modulus, which turns out to be nearly double [8]. Consequently, the modulus contrast between the two phases should be detectable by nanoindentation. The following approach has been used for both materials:

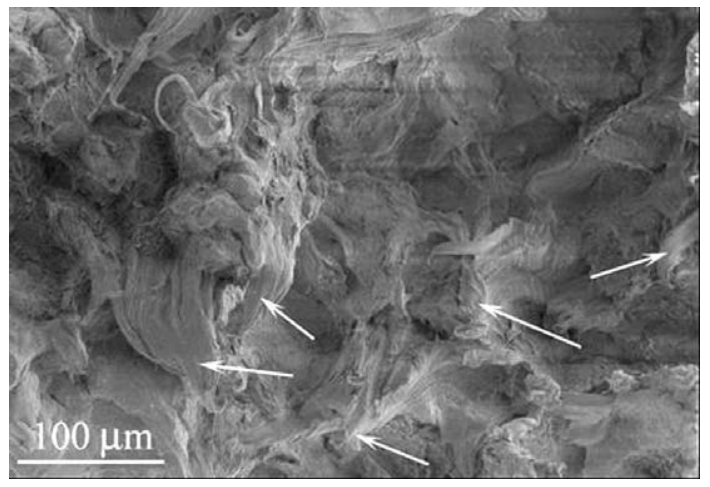

Fig. 7. Fracture surface of HVC UHMWPE. Very high recrystallized phase fraction $\left(f_{R} \sim 80 \%\right)$. 
- an indentation series was first performed on a melt-crystallized sample to evaluate local variations within a single phase and experimental uncertainty;

- the same experiment was performed on a HVC sample;

- the indent marks on the HVC sample were observed by optical microscopy and their image was displayed superimposed on the indentation results to locate the stiff and soft regions.

A series of 50 indents on a straight line was examined. Indents were made every $10 \mu \mathrm{m}$ in such a way that a line of $490 \mu \mathrm{m}$ is probed, which is several times larger than the average particle size (around $100 \mu \mathrm{m}$ ).

First, the optical micrographs of indented surfaces are presented and analyzed, and then the elastic moduli from indentation experiments are superimposed on the corresponding micrographs to locate the different phases. The indent marks are clearly visible on the HVC POM sample, as seen in Fig. 8. However, only rare incompletely welded interfaces can help to distinguish the original particles. It should be noted that examination at higher magnification has shown that the large black marks correspond to dust or scratches and not to porosities.

On the micrograph of HVC UHMWPE (Fig. 9) a significant contrast is present and particles are clearly visible, but the indent marks are difficult to distinguish within the dark zones. However, the use of Nomarski contrast mode at the highest magnification enabled the indents always to be located.

Before analysis of indentation data, the origin of the contrast observed on HVC UHMWPE samples will be discussed. Cryoultramicrotomy surfacing is believed to produce extremely flat surfaces that would reflect light and appear bright under optical microscope (this is actually what is observed on melt-crystallized and HVC POM samples). Consequently, dark regions could reveal a more significant relief that would reflect less light. This relief difference has been confirmed by AFM observation: it is seen in Fig. 10 that the left region, which appears dark, presents a higher relief. In addition, height and lateral dimensions of the bumps in the dark zone are of the same order

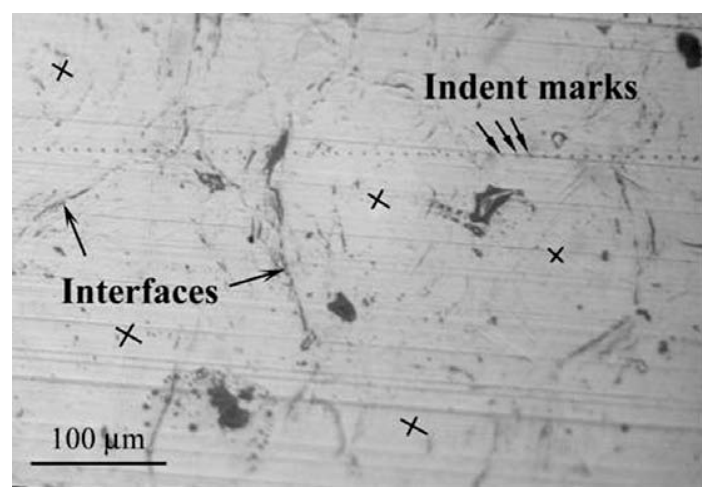

Fig. 8. Optical micrograph of HVC POM. Recrystallized phase fraction $f_{R} \sim 20 \%$. Crosses indicate nascent particles.

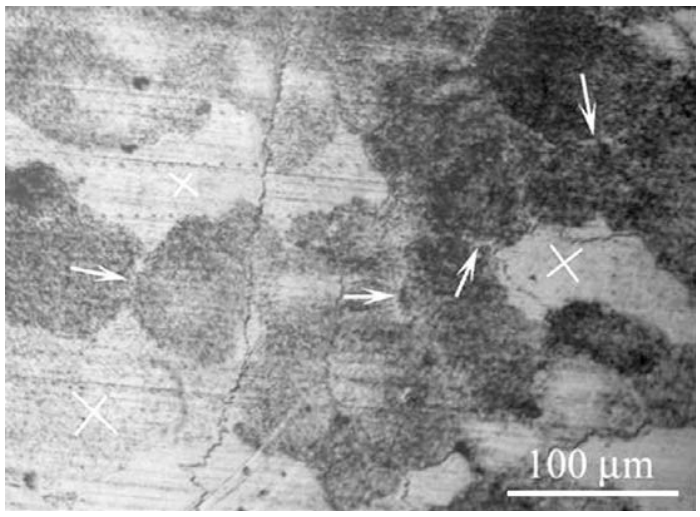

Fig. 9. Optical micrograph of HVC UHMWPE. Recrystallized phase fraction $f_{R} \sim 50 \%$. White arrows indicate recrystallized interfaces. Crosses indicate large melted-recrystallized zones. The black line going from top to bottom is an artefact due to microtomy.

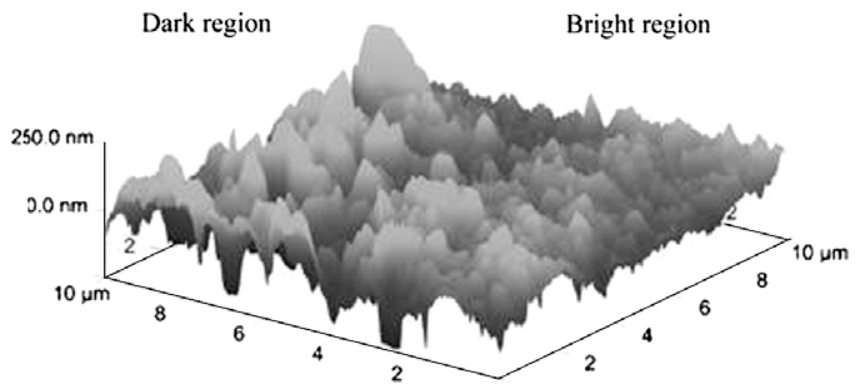

Fig. 10. AFM height image obtained in standard tapping mode showing the relief difference between dark and bright regions.

of magnitude as the light wavelength, confirming that interactions between light and surface are possible.

Nascent UHMWPE is suspected to have considerable internal stresses created during simultaneous polymerization and crystallization [14]. Therefore, one possible explanation for the observed relief would be that these internal stresses relax after microtomy to create micron-sized bumps. In this case, the relief would not appear in recrystallized zones, and dark zones would reveal the presence of nascent material.

Additional optical microscopy observations have been conducted on HVC UHMWPE samples with different fractions of recrystallized phase to confirm this hypothesis. In Fig. 11, it is observed that a sample with a low recrystallized phase fraction (determined by DSC) effectively exhibits mainly dark zones. It is of great practical interest to obtain an insight into the fraction of recrystallized material and the spatial distribution of the two phases. Considering the hypothesis that the recrystallized phase appears brighter, two types of interfaces are observed within HVC UHMWPE samples: open interfaces (i.e. interparticle voids) appearing black, and recrystallized interfaces appearing white. It is noteworthy that while at low recrystallized phase fraction (Fig. 11) there are numerous open interfaces, at higher fractions of recrystallized phase (Fig. 9) these have almost disappeared. In Fig. 9, i.e. at high $f_{R}$, it is observed that there are 


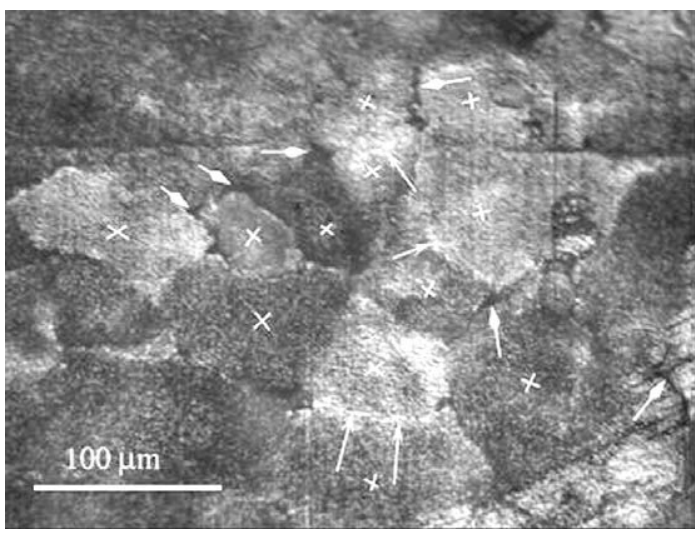

Fig. 11. Optical micrograph of HVC UHMWPE. Recrystallized phase fraction $f_{R} \sim 5 \%$. Thick arrows indicate open interfaces. Thin arrows indicate recrystallized interfaces. Crosses indicate nascent particles.

large recrystallized zones, which is consistent with the thick fibrils observed on fracture surfaces from $f_{R} \sim 50 \%$.

To confirm conclusively the presence of two phases with different crystallinities, and hence different moduli, local mechanical properties from nanoindentation experiments are presented. The local elastic moduli are displayed superimposed on the corresponding optical micrographs (Figs. 12 and 13). The elastic moduli obtained on melt-crystallized material are presented with their mean value $\mu$ and their standard deviation $\sigma$. It appears that HVC material has local mechanical properties that are strongly heterogeneous with Young's moduli varying from 4.6 to $9.9 \mathrm{GPa}$ for $\mathrm{HVC} \mathrm{POM}$ and from 1.2 to $2.2 \mathrm{GPa}$ for $\mathrm{HVC}$ UHMWPE. High elastic modulus regions can be attributed to nascent phase and low elastic modulus ones to meltedrecrystallized phase. As already evoked in the experimental section, local elastic moduli from nanoindentation are usually higher than conventionally measured macroscopic ones and cannot be compared. However, the ratio of $\sim 2$ between nascent and melt-crystallized elastic moduli esti-

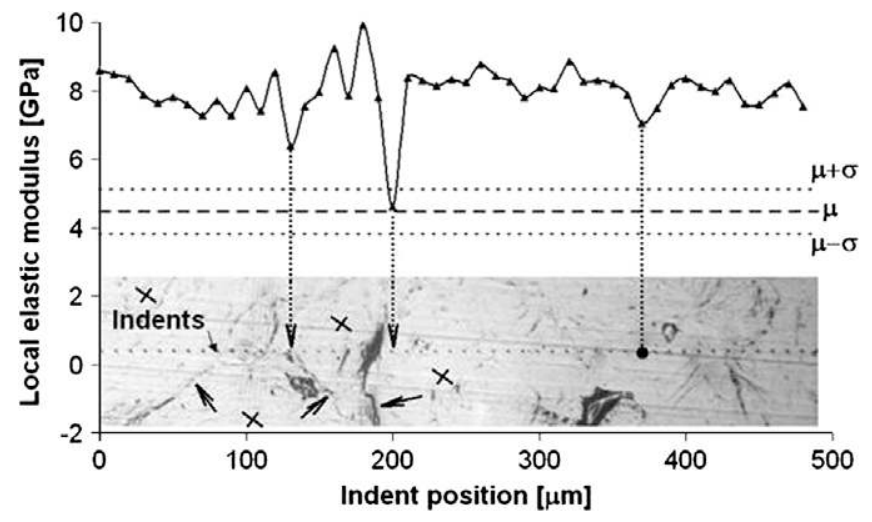

Fig. 12. Combined optical microscopy and nanoindentation of HVC POM (plain line). $\mu$ and $\sigma$ are the mean value and the standard deviation of melt-crystallized sample local Young's moduli (dotted lines). Crosses indicate nascent particles. Short arrows Crosses indicate interfaces. Long arrows indicate recrystallized phase at particle interfaces. Plain circle indicates softer zone within nascent particle.

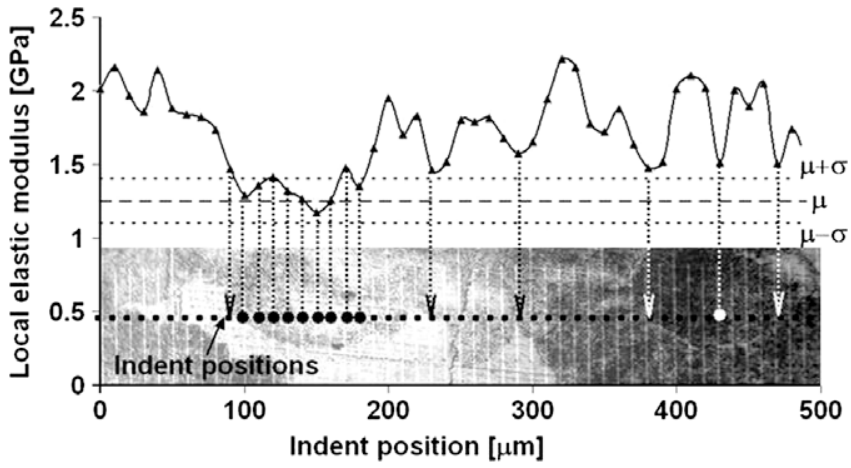

Fig. 13. Combined optical microscopy and nanoindentation of HVC UHMWPE (plain line). $\mu$ and $\sigma$ are the mean value and the standard deviation of the melt-crystallized sample local Young's moduli (dotted lines). Plain circles indicate softer zones within nascent particles.

mated in our previous work [8] from macroscopic mechanical tests is found again here.

The combined analysis of optical micrographs and nanoindentation results shows that the recrystallized phase is both located at particle interfaces (indicated by arrows in Figs. 12 and 13) and also within nascent particles (indicated by circles in Figs. 12 and 13). It is also noted that intermediate moduli have been measured (e.g. between 200 and $300 \mu \mathrm{m}$ for HVC UHMWPE). As shown by finite-element analysis $[15,16]$, the local moduli obtained by nanoindentation are influenced by a large zone around the indents, which makes the interpretation of these intermediate moduli difficult. They could be attributed to zones with effectively intermediate moduli or originate in the presence of recrystallized material close enough to the indent to influence the local elastic modulus value.

In the particular case of UHMWPE, nanoindentation measurements are only partially consistent with the previous hypothesis that the nascent phase appears dark and the recrystallized phase bright. This could result from:

- the influence of the indent environment in the three directions (e.g. an indentation in a soft zone close to a stiff zone would result in an overestimated modulus $[15,16])$;

- possible modulus heterogeneities inside the nascent particles: even if nascent UHMWPE is globally more crystalline, the presence of low-crystallinity zones cannot be excluded. These zones would appear dark as the optical contrast is not related to the crystallinity but originates from the internal stresses of nascent UHMWPE.

The experiments carried out in this first section show that some, but not all, of the recrystallized phase is located at particle interfaces. In particular, at high recrystallized phase fractions, it appears that some particles have been completely melted-recrystallized while others have been kept at the nascent state and only exhibit surface melting-recrystallization. This leads us to conclude that melting 
due to self-heating under HVC impacts would first take place at interfaces but then would also occur within nascent particles. Located at the interfaces, the melting/recrystallization of nascent polymer should strongly contribute to the welding of the particles.

\section{Discussion}

\subsection{Review of the different possible sintering mechanisms}

In light of existing work on the subject, different sintering mechanisms are considered, before and after the local melting.

Molecular interaction forces (Van der Waals) in amorphous phase are too low to provide strong cohesion, and the strength of structural polymers originates in entanglements and crystallites that link macromolecules together [17]. Consequently, a good sintering can only be provided by the creation of entanglements or crystallites that can connect molecules from adjacent powder particles.

As UHMWPE and POM are highly crystalline polymers, particularly at the nascent state, sintering by diffusion and intertwining of amorphous chains is likely to be impossible before melting of the crystallites. Indeed, highly crystalline polymer powders offer very few opportunities for particle sintering through amorphous-amorphous interfaces of neighbour particles. In addition, some of the amorphous phase is formed of chains also belonging to one or more crystallites, thus with limited diffusion possibilities.

However, crystallographic welding (either by epitaxial coherence [18] or by lamellae doubling [19]), promoted by high crystal mobility near the melting temperature and high pressures, would be possible before local melting. Chain diffusion within the crystal is suspected to play an important role in crystal matching. Therefore, this kind of sintering is assumed to be a very slow mechanism. It could occur in the first stage of HVC, but we believe that the processing time $(\sim 1 \mathrm{~min})$ is too short to obtain a good sintering by crystallographic welding. It is consistent with the fact that it has not been possible to obtain efficient welding (measurable mechanical properties) of the particles without partial melting.

This analysis shows that significant sintering could not occur during HVC without local melting and subsequent recrystallization. Ward et al. have successfully welded polymer fibers by significant local melting at the surface of the fibers followed by recrystallization [20]. Similarly, in the case of polymers processed by HVC, it is thought that nascent particles are welded by a melting and recrystallization that appears first at particle interfaces.

The melting point would be reached locally by self-heating under HVC impacts. During the first 10 impacts, selfheating is probably caused by viscoelastic and viscoplastic energy dissipation due to significant confined compressive elastic and plastic deformation under the impacts. After that, since the sample is fully dense, self-heating is only caused by viscoelastic energy dissipation which is believed to originate both from molecular motions (classical interpretation of viscoelastic phenomena in polymers [10]) and friction between non-welded particles. This last phenomenon would cause localized self-heating at particle interfaces and therefore could explain why melting first occurs there.

The amount of melted polymer increases with HVC impacts as schematized in Fig. 14 and governs the microstructure of the final material. At low $f_{R}(\sim 20 \%)$, the material is fully dense and the particles are welded together by the recrystallized phase. At higher $f_{R}$, it has been observed that the recrystallized phase can be located also within the nascent particles. It is assumed that the recrystallized phase will percolate at a certain fraction to form a continuous matrix where small regions of nascent material would act as reinforcing domains (Fig. 14).

When local melting within the interfaces occurs, two principal mechanisms can be involved at the molecular scale to provide welding between the particles: diffusion of the chains across the interfaces and recrystallization of these chains. Therefore, after recrystallization, there is a high probability of finding chains coming from two adjacent powder particles in crystal lamellae located at the interface. This mechanism (called co-crystallization) will provide strong links between the particles. Several studies on semicrystalline welding have shown that co-crystallization significantly contributes to the strength of a welded interface $[21,22]$. The formation of entanglements and cocrystallites at the interface has been sketched in Fig. 15.

\subsection{Sintering quality}

It is now proposed to discuss the strength of the local welds and then the resulting quality of the overall sintering.

\subsubsection{Efficiency of the welding at the molecular scale}

Chain diffusion implies large-scale molecular motions. For equilibrated melts, these motions are well described by the reptation theory $[23,24]$. Just after melting, an equilibrated melt state is easily obtained for low-viscosity polymer (e.g. POM), but not for high-viscosity polymers (e.g. UHMWPE). Moreover the nascent structures $[9,25]$ are often disentangled as they are out of equilibrium; however. the UHMWPE powder used here is believed to be partially entangled $[25,26]$. Whatever the state of the melt polymer, the main issue is the ability for the chains to diffuse across the interface between powder particles to obtain a sintered material. In a first approximation, to evaluate the diffusion at the interface, the reptation model is used for both POM and UHMWPE. The reptation time $t_{\text {rep }}$ is given by the following equation [24]:

$t_{\text {rep }}=\tau_{1} \frac{N^{3}}{N_{e}}$

where $N$ is the number of monomer units per chain, $N_{e}$ is the number of monomer units between entanglements, and $\tau_{1}$ is the microscopic time of monomer motion. This 


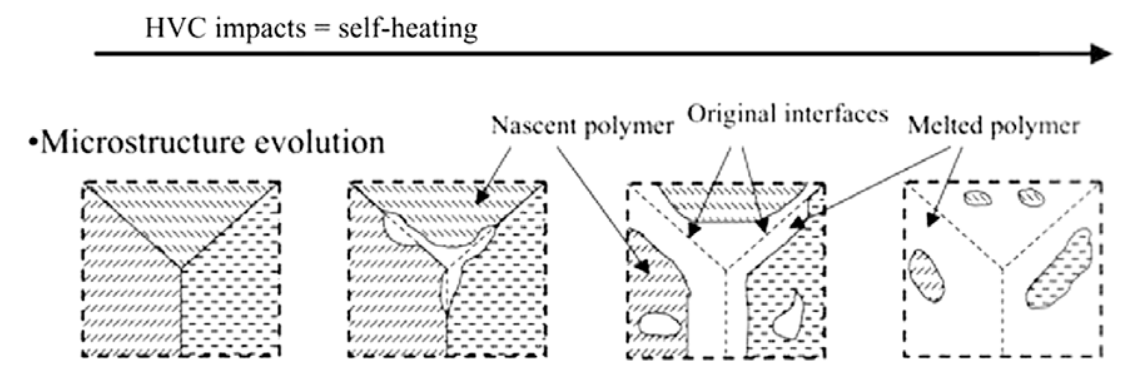

-Corresponding failure surfaces

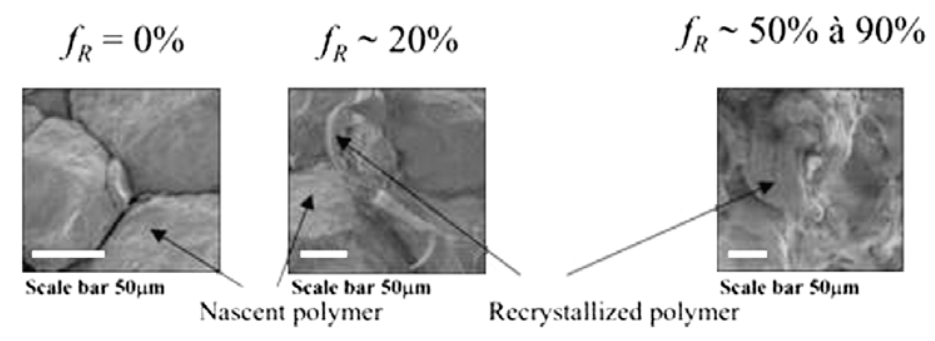

Fig. 14. Scenario of the sintering mechanism involved in HVC.

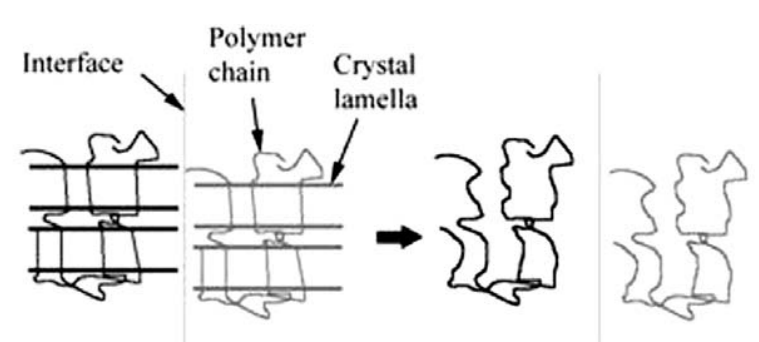

Initial configuration
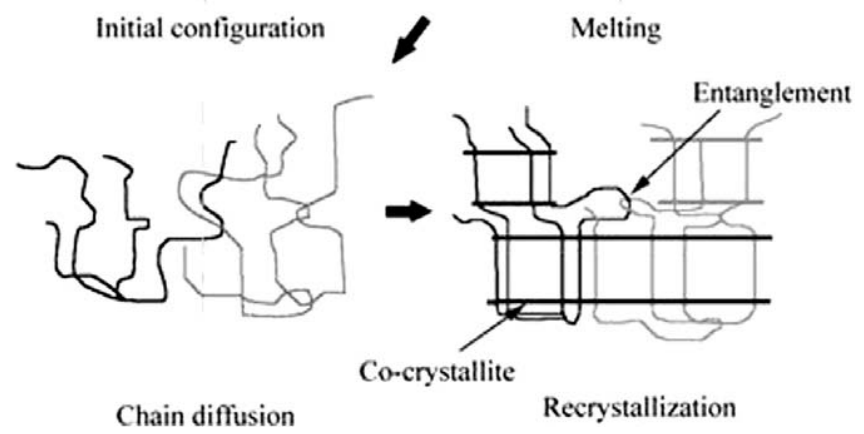

Fig. 15. Two-dimensional schematic representation of the welding mechanisms at the molecular scale.

time is around $10^{-10} \mathrm{~s}$ just above the melting temperature for PE and POM $[23,27,28]$. Using the reptation theory, Wool et al. $[29,30]$ have calculated the so-called average interpenetration distance at a polymer-polymer interface $\chi$ in order to model polymer welding. They demonstrated the following scaling law:

$\chi(t)=R_{g}\left(\frac{t}{t_{\text {rep }}}\right)^{1 / 4}$

where $R_{g}$ is the radius of gyration given by [24]:

$R_{g}=b_{o} \sqrt{C_{\infty} \frac{N}{6}}$ where $b_{\mathrm{o}}$ is the length bond and $C_{\infty}$ is the characteristic ratio representing the flexibility of the chain. $C_{\infty} \sim 6$ for PE and $C_{\infty} \sim 7.5$ for POM [31], using $N=1500$ and $N_{e}=67$ for POM [7,32] and $N=280000$ and $N_{e}=89$ for UHMWPE $[8,32]$. POM reptation time is approximately $0.01 \mathrm{~s}$, while the UHMWPE reptation time is more than 20,000 s due to its very high N. It follows that interpenetration is considerably more difficult within UHMWPE than within POM. The interpenetration distance $\chi$ has been plotted as a function of time in Fig. 16 for POM and UHMWPE.

During HVC, particle interfaces would be maintained at the melt state for approximately $60 \mathrm{~s}$. It follows that this time is largely sufficient to achieve complete erasure of the interface for POM as $\chi$ is much higher than $R_{\mathrm{g}}$ (perfect sintering criterion: $\chi=R_{\mathrm{g}}$ Conversely, for UHMWPE, a period of $60 \mathrm{~s}$ only allows partial interpenetration. The very long diffusion time of UHMWPE has been previously underlined by Buckley et al. who demonstrated that diffusion defects remain even after the $2 \mathrm{~h}$ sintering process time

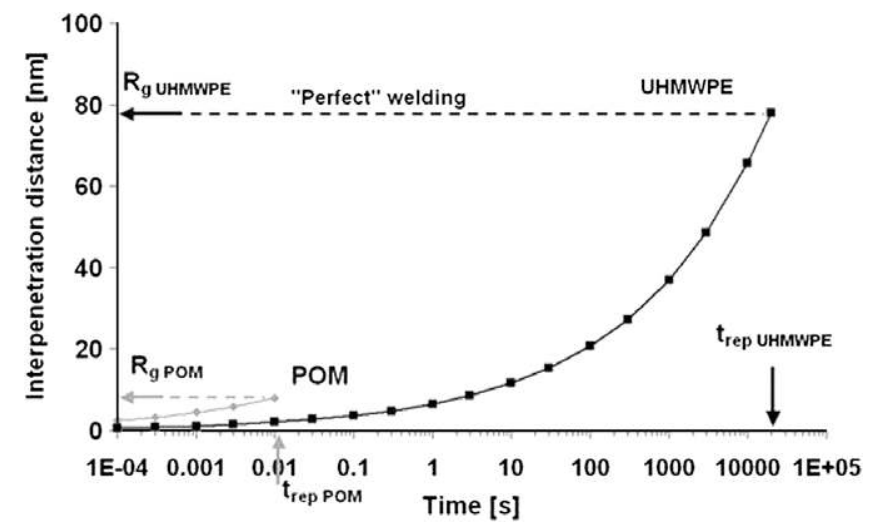

Fig. 16. Average interpenetration distance at the interface from reptation theory. 
generally used [33,34]. A complete erasure of the initial interfaces would be achieved only for a polymer with a short reptation time (i.e. an injectable grade). However, it should be emphasized that several phenomena not taken into account in the Wool interpenetration model could accelerate chain diffusion for the HVC process. It is, for instance, thought that the volume expansion during melting would promote chain diffusion across the interface. Moreover, for the out-of-equilibrium nascent structure of UHMWPE, chains can drastically increase their volume just after melting to recover their equilibrium configuration [35]. This phenomenon, called "chain-explosion", should favour interpenetration.

Considering only the interpenetration distance (probably greatly underestimated) obtained by the reptation model, Fig. 16 shows that after $60 \mathrm{~s}$ an interpenetration distance of $20 \mathrm{~nm}$ is attained for UHMWPE, which would be adequate to create strong links by co-crystallization during cooling. It is thought that due to this co-crystallization, large diffusion is not absolutely required to obtain strong welding. Therefore, even if the complete erasure of the interfaces is not reached within HVC UHMWPE, it is believed that HVC provides strong welds between nascent particles by co-crystallization.

\subsubsection{Overall sintering quality}

Material mechanical properties, in particular the fracture strain, provide an insight into the overall sintering quality. Fracture strains of UHMWPE from tensile tests at $0.001 \mathrm{~s}^{-1}$ obtained in previous work $[8,16]$ have been plotted as a function of $f_{R}$ in Fig. 17. For POM, results from mechanical tests at $130{ }^{\circ} \mathrm{C}$ are reported. Testing at high temperature avoids premature failure due to intrinsic brittleness of POM nascent particles.

Significant ductility (i.e. $30 \%$ to more than $100 \%$ true strain) is obtained on HVC samples with at least $50 \%$ of recrystallized phase, while below $f_{R} \sim 30 \%$, samples are brittle (i.e. they exhibit less than $10 \%$ true strain). HVC impact pressure (several hundred MPa [36]) leads to nearly

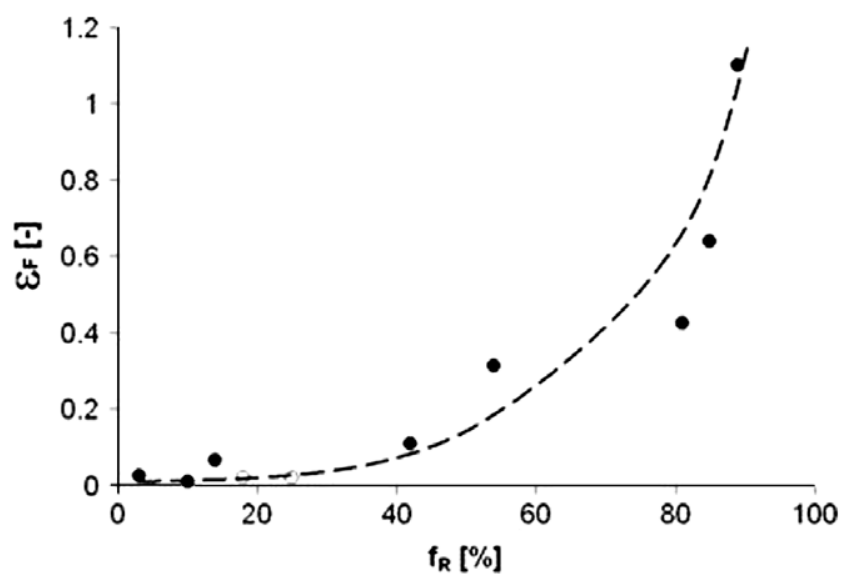

Fig. 17. Fracture strain $\varepsilon_{\mathrm{F}}$ vs. recrystallized phase fraction $f_{R}$ from tensile tests. Filled symbols indicate HVC UHMWPE tensile tests at $20^{\circ} \mathrm{C}$ [8]. Open symbols indicate HVC POM tensile tests at $130{ }^{\circ} \mathrm{C}$ [16]. complete densification of the material [7,8]. However, as seen in Figs. 10 and 12, some localized interparticle voids are present at low $f_{R}$. In addition, a close examination of Fig. 7 shows that fibrils do not cover the whole particle surfaces, thus it is suspected that a significant part of the interfaces, even if in close contact, have not been meltedrecrystallized. It follows that a partial stress transmission leads to an early breaking of the sample. These brittle samples exhibit higher strains during compressive testing [7], which reinforce the idea that unwelded interfaces exist at low $f_{R}$ as the compressive stress state reduces the effects of sintering defects. The brittleness at low $f_{R}$ is thus attributed to the presence of numerous "unwelded" interfaces. As schematized in Fig. 14, the increasing fraction of recrystallized phase leads to more and more welded interfaces and thus higher fracture strains. It follows that for a given polymer, the overall sintering quality is governed by the fraction of recrystallized material. It can be also assumed that the strong increase in the fracture strain around $f_{R}=50 \%$ can be explained by the percolation of the recrystallized part.

\section{Conclusion}

Two nascent highly crystalline polymer powders have been successfully processed by the new HVC technique via sintering $[7,8]$. It is assumed that $\mathrm{HVC}$ impacts provoke self-heating within the sample, initially at particle interfaces due to friction between the particles. The melting temperature of the nascent polymer would thus be attained locally in this zone. Within melted interfaces the sintering would then occur by diffusion and intertwining of the chains and by co-crystallization during cooling. This sintering scenario could be universal for all semicrystalline polymers.

Considering the reptation theory, it has been shown that HVC allows the complete diffusion of the chains for classical molecular weight polymers. On the contrary, ultra-high molecular weight polymers such as UHMWPE would only partially diffuse, but the interpenetration of the chains would be sufficient to obtain co-crystallization and therefore a good welding of the melted interfaces.

The overall sintering quality seems to be governed by the fraction of recrystallized material. At low $f_{R}$, the melting would not be achieved within all interfaces, thus leaving unwelded particles. Therefore a limited fracture strain is observed for these samples due to stress concentrations that initiate fast cracking. A high $f_{R}$ is needed to obtain a significant ductility, which has been possible for UHMWPE but not for POM, due to polymer flowing at ejection and thermochemical degradation above $f_{R} \sim 20 \%$.

These results are highly instructive in terms of extending HVC to other polymers. (A promising study has shown that PTFE can be processed by HVC [37].) It is worth noting that it would be conceivable to extend HVC to amorphous polymers with a low reptation time, as complete diffusion would be achieved at melted interfaces.

Regarding very high molecular weight polymers, due to the low diffusion rate, chain diffusion defects remain within 
the HVC material. However, a strong welding of the interfaces would be provided by co-crystallization and therefore the use of semicrystalline polymers is likely to be essential in this case.

\section{References}

[1] Parasnis NC, Ramani K. J Mater Sci: Mater Med 1998;9:165.

[2] Kelly JM. J Macromol Sci: Part C Polym Rev 2002;42:355.

[3] Andena L, Rink M. Polym Eng Sci 2004;44:1368.

[4] Hambir SS, Jog JP, Nadkarni VM. Polym Eng Sci 1994;34:1065.

[5] Al Jebawi K, Sixou B, Seguela R, Vigier G, Chervin C. J Appl Polym Sci 2006;102:1274.

[6] Myasnikova LP, Egorov EA, Zhizhenkov VV, Kvachadze NG, Boiko YM, Ivan'kova EM, et al. Polym Sci Ser A 2008;50:640.

[7] Jauffrès D, Lame O, Vigier G, Doré F, Chervin C. J Appl Polym Sci 2007;106:488.

[8] Jauffrès D, Lame O, Vigier G, Doré F. Polymer 2007;48:6374.

[9] Rastogi S, Lippits DR, Hohne GW, Mezari B, Magusin PCMM. J Phys: Condens Mat 2007;19:205122.

[10] Ward IM, Sweeney J. Mechanical properties of solid polymers. London: Wiley; 2004.

[11] Labour T, Ferry L, Gauthier C, Hajji P, Vigier G. J Appl Polym Sci 1999;74:195.

[12] Van Landingham MR, Villarrubia JS, Guthrie WF, Meyers GF. Macromol. Symp. 2001;167:15.

[13] Jauffrès D, Lame O, Vigier G, Fridrici V, Doré F. J Appl Polym Sci 2007;110:2579.

[14] Morin FG, Delmas G, Gilson DFR. Macromolecules 1995;28: 3248.

[15] Lee SH, Wang S, Pharr GM, Xu H. Composites Part A: Appl Sci Manuf 2007;38:1517.
[16] Gauthier C, Chazeau L, Gaertner R. Gordon Research Conference, Ventura, CA, 2002.

[17] Mark JE. Physical properties of polymers. Washington, DC: American Chemical Society; 1993.

[18] Al Jebawi K, Sixou B, Seguela R, Vigier G. J Appl Polym Sci 2007;106:757.

[19] Rastogi S, Spoelstra AB, Goossens JGP, Lemstra PJ. Macromolecules 1997;30:7880.

[20] Ward IM, Hine PJ. Polymer 2004;45:1413.

[21] Bonten C, Schmachtenberg E. Polym Eng Sci 2001;41:475.

[22] Xue YQ, Tervoort TA, Rastogi S, Lemstra PJ. Macromolecules 2000;33:7084

[23] De Gennes PG, Leger L. Annu Rev Phys Chem 1982;33:49.

[24] Doi M, Edwards SF. The theory of polymer dynamics. Oxford: Oxford University Press; 1986.

[25] Rastogi S, Lippits DR, Peters GWM, Graf R, Yao Y, Spiess HW. Nat Mater 2005;4:635.

[26] Jauffrès D, Lame O, Vigier G, Doré F. Macromolecules 2008;41: 9793.

[27] Robelin-Souffaché E, Rault J. Macromolecules 1984;17:337.

[28] Porter CH, Lawler JH, Boyd RH. Macromolecules 1970;3:308.

[29] Kim YH, Wool RP. Macromolecules 1983;16:1115.

[30] Wool RP, Yuan BL, McGarel OJ. Polym Eng Sci 1989;29:1340.

[31] Zhang LX, Xia A, Zhao DL. J Polym Sci: Part B: Polym Phys 2000;38:3163.

[32] Ferry JD. Viscoelastic properties of polymers. 3rd ed. New York: Wiley; 1980.

[33] Buckley CP, Wu J, Haughie DW. Biomaterials 2006;27:3178.

[34] Haughie DW, Buckley CP, Wu J. Biomaterials 2006;27:3875.

[35] De Gennes PG. Comptesrendus de l'Académie des Sciences. Série II 1995;321:363.

[36] Azhdar B, Stenberg B, Kari L. Polym Test 2005;24:909.

[37] Sriti I, Master's thesis, INSA-LYON, Lyon; 2003. 